Homology, Homotopy and Applications, vol.10(2), 2008, pp.135-137

\title{
PERIODIC COHOMOLOGY
}

\author{
W.H. MANNAN \\ (communicated by Graham Ellis)
}

\begin{abstract}
We remark that a very short proof of an elementary result concerning cohomological periods is implicit in the existing literature. As a corollary we show that given a finitely generated stably free resolution of the integers over a finite group, two of its modules are free.
\end{abstract}

\section{Introduction}

The study of periodic cohomology over finite groups closely relates to free group actions on spheres (see, for example, $[\mathbf{3}]$ ). This in part contributes to the importance of the classification of finite groups with periodic cohomology (see [4] and [6]).

A much simpler classification is the classification of groups which specifically have cohomological period 2. They must be cyclic. Although well-known, the proof in the literature (see [5]) is disproportionately technical, involving the methods used in more general classifications of periodic cohomology. This includes considering potential Sylow $p$-subgroups and finding normal complements. We deduce a direct proof from the characterization of periodic cohomology in [1]. This avoids such technical difficulties.

In $\S 2$ we use this result to prove a result regarding finite stably free resolutions of $\mathbb{Z}$ over finite groups:

Theorem A. Let $G$ be a finite group and suppose we have a finitely generated stably free resolution over $G$ :

$$
\cdots \stackrel{d_{3}}{\rightarrow} S_{2} \stackrel{d_{2}}{\rightarrow} S_{1} \stackrel{d_{1}}{\rightarrow} S_{0} \rightarrow \mathbb{Z}
$$

Then $S_{1}$ and $S_{2}$ are free.

The chain complex associated to the universal cover of a cell complex may be regarded as an algebraic complex over the fundamental group. In particular, if the cell complex is finite and 2-dimensional, then one obtains a truncated free finite resolution of $\mathbb{Z}$. The classification of these homotopy types is of particular interest due to its relation to Wall's D2 problem (see the introduction to [1]).

Although the modules in these complexes are free, algebraic surgery may leave some modules potentially only stably free. From the point of view of classification, it

Received November 24, 2007; published on August 21, 2008.

2000 Mathematics Subject Classification: 16E05.

Key words and phrases: projective resolution, periodic homology.

This article is available at http://intlpress.com/HHA/v10/n2/a6

Copyright (C) 2008, International Press. Permission to copy for private use granted. 
is useful to know that these modules will still be free (in particular, as the maps may then be represented by matrices). Over finite groups, Theorem A does just that.

\section{Cohomological period}

We follow the characterization of cohomological period in [1] by saying that $n>0$ is a (cohomological) period of a finite group $G$ if one of the following equivalent conditions holds (see $[\mathbf{1}, \S 40], \mathcal{P}_{1}(n), \mathcal{P}_{3}(n), \mathcal{P}_{4}(n)$ ):

$C_{1}(n): \mathcal{D}_{n+k}(\mathbb{Z}) \cong \mathcal{D}_{k}(\mathbb{Z})$ for all integers $k$. $\left(\mathcal{D}_{n}\right.$ denotes the $n^{\text {th }}$ iteration of Johnson's "derived functor", defined in $[\mathbf{1}, \S 20]$. In particular, $\widehat{H}^{a n+k}(G ; \mathbb{Z}) \cong$ $\widehat{H}^{b n+k}(G ; \mathbb{Z})$ for all $a, b, k \in \mathbb{Z}$.)

$C_{2}(n)$ : There exists an exact sequence of the form

$$
0 \rightarrow \mathbb{Z} \rightarrow P_{n-1} \rightarrow \cdots \rightarrow P_{0} \rightarrow \mathbb{Z} \rightarrow 0,
$$

where the $P_{i}, i=0, \ldots, n-1$, are projective.

$C_{3}(n): \widehat{H}^{n}(G ; \mathbb{Z}) \cong \mathbb{Z} /|G|$.

Note that $\mathcal{C}_{1}(n)$ implies $H_{n-1}(G ; \mathbb{Z}) \cong \widehat{H}^{-n}(G ; \mathbb{Z}) \cong \widehat{H}^{n}(G ; \mathbb{Z})$. Hence, if $n$ is a period of $G$, then $H_{n-1}(G ; \mathbb{Z}) \cong \mathbb{Z} /|G|$.

Theorem 2.1. If 2 is a period of a finite group $G$, then $G$ is cyclic.

Proof. $G / G^{\prime} \cong H_{1}(G ; \mathbb{Z}) \cong \mathbb{Z} /|G|$, which has the same order as $G$ so $G \cong G / G^{\prime}$.

\section{Stably free resolutions}

Before using Theorem 2.1 to prove Theorem A, we note the following restriction on stably free modules over finite groups:

Proposition 3.1. Any stably free module of finite $\mathbb{Z}[G]$-rank greater than one is free.

Proof. $\mathbb{Z}[G] \oplus \mathbb{Z}[G]$ is an Eichler lattice so the result follows from [1, Thm. 15.1].

Proof of Theorem A. Suppose one of $S_{1}$ or $S_{2}$ is not free and let $K=\operatorname{ker}\left(d_{1}\right)$. We have an exact sequence:

$$
0 \rightarrow K \rightarrow S_{1} \stackrel{d_{1}}{\rightarrow} S_{0} \rightarrow \mathbb{Z} \rightarrow 0 .
$$

Consideration of ranks and nullities implies $\operatorname{rk}_{\mathbb{Z}}(K) \equiv 1 \bmod |G|$.

$K$ is a submodule of $S_{1}$ and $d_{2}$ induces a surjection $S_{2} \rightarrow K$. Hence,

$$
\operatorname{rk}_{\mathbb{Z}}(K) \leqslant \operatorname{rk}_{\mathbb{Z}}\left(S_{1}\right), \operatorname{rk}_{\mathbb{Z}}\left(S_{2}\right) .
$$

By Proposition 3.1, we have $\operatorname{rk}_{\mathbb{Z}}\left(S_{1}\right) \leqslant|G|$ or $\operatorname{rk}_{\mathbb{Z}}\left(S_{2}\right) \leqslant|G|$, so either way $\operatorname{rk}_{\mathbb{Z}}(K)=1$.

Tensoring (1) with $\mathbb{Q}$ yields the exact sequence

$$
0 \rightarrow K \otimes \mathbb{Q} \rightarrow \mathbb{Q}[G]^{a} \rightarrow \mathbb{Q}[G]^{b} \rightarrow \mathbb{Q} \rightarrow 0 .
$$

By the "Whitehead Trick", $K \otimes \mathbb{Q} \oplus \mathbb{Q}[G]^{b} \cong \mathbb{Q} \oplus \mathbb{Q}[G]^{a}$ as $\mathbb{Q}[G]$ - modules. Consideration of dimension over $\mathbb{Q}$ implies $a=b$, so cancelation gives $K \otimes \mathbb{Q} \cong \mathbb{Q}$. In particular, the $G$-action on $K \otimes \mathbb{Q}$ is trivial. Hence the $G$-action on $K$ is trivial and we 
have $K \cong \mathbb{Z}$. We therefore have an exact sequence

$$
0 \rightarrow \mathbb{Z} \rightarrow S_{1} \stackrel{d_{1}}{\rightarrow} S_{0} \rightarrow \mathbb{Z} \rightarrow 0 .
$$

Hence $G$ satisfies $\mathcal{C}_{2}(2)$ and is cyclic by Theorem 2.1 .

Any finitely generated stably free module over a cyclic group is free (see $[\mathbf{1}$, Proposition 15.7]). So, in particular, $S_{1}$ and $S_{2}$ are free as required.

Note, however, that $S_{0}$ need not be free. For example, if $G$ is the quaternionic group $Q_{32}$, then we have a finitely generated stably free resolution where $S_{0}$ is not free $($ see $[\mathbf{2}, \S 4])$.

\section{References}

[1] F.E.A. Johnson, Stable modules and the $\mathrm{D}(2)$-problem, London Mathematical Society Lecture Note Series 301, Cambridge University Press, Cambridge, 2003.

[2] F.E.A. Johnson, Minimal 2-complexes and the $\mathrm{D}(2)$-problem, Proceedings of the American Mathematical Society 132, no. 2 (2003), 579-586.

[3] J. Milnor, Groups which act on $S^{n}$ without fixed points, American Journal of Mathematics 79 (1957), 623-630.

[4] M. Suzuki, On finite groups with cyclic Sylow subgroups for all odd primes, American Journal of Mathematics 77 (1965), 657-691.

[5] R.G. Swan, Minimal resolutions for finite groups, Topology 4 (1965), 193-208.

[6] H. Zassenhaus, Über endliche Fastkörper, Abhandlungen aus dem Mathematischen Seminar der Hamburgischen Universität 11 (1935), 187-220.

W.H. Mannan wajid@mannan.info

J8 Hicks Building, Department of Pure Mathematics, University of Sheffield, Hounsfield Road, Sheffield S3 7RH, United Kingdom 